\title{
Occlusion of the Celiac Artery during Endovascular Thoracoabdominal Aortic Aneurysm Repair Is associated with Increased Perioperative Morbidity and Mortality
}

\author{
Ryan W. King ${ }^{1}$, Ryan Gedney ${ }^{2}$, Jean Marie Ruddy ${ }^{1,3}$, Elizabeth A. Genovese ${ }^{1,3}$, Thomas E. \\ Brothers $^{1,3}$, Ravi K. Veeraswamy ${ }^{1}$, Mathew D. Wooster ${ }^{1}$ \\ ${ }^{1}$ Division of Vascular Surgery, Medical University of South Carolina, Charleston, SC. \\ ${ }^{2}$ College of Medicine, Medical University of South Carolina, Charleston, SC. \\ ${ }^{3}$ Ralph H. Johnson Department of Veterans Affairs Medical Center, Charleston, SC.
}

\section{Abstract}

Background: Some studies suggest that celiac artery coverage during elective endovascular thoracoabdominal aortic aneurysm (TAAA) repair is safe given sufficient collateralization of visceral organ perfusion from the superior mesenteric artery. However, there is concern that celiac artery coverage may lead to increased risk of foregut or spinal cord ischemia with an attendant increased risk of mortality. We sought to investigate rates of bowel ischemia, spinal cord ischemia, and 30-day mortality associated with celiac artery coverage during TEVAR and complex EVAR.

Methods: The Society for Vascular Surgery Vascular Quality Initiative database was queried for TEVAR and complex EVAR cases from 2012 to 2018. Inclusion criteria included TAAA pathology and endograft extension to aortic zone 6. Patients with aortic rupture, trauma, prior thoracic aortic surgery, known preoperative occlusion of the left subclavian superior mesenteric, or celiac arteries were excluded. Cases with intraoperative celiac artery occlusion (CAO) were compared retrospectively to cases with celiac artery preservation (CAP). Primary outcomes included 30-day mortality and a composite end point of 30-day mortality, spinal cord ischemia (transient or permanent lower extremity neurologic deficit), and bowel ischemia (colonoscopic evidence of ischemia, bloody stools in a patient who dies prior to colonoscopy or laparotomy, or other documented clinical diagnosis). Univariable comparisons were performed using chisquared tests and Student's $t$-tests, as appropriate. Multivariable logistic regression analyses were employed to identify independent predictors of outcome.

Results: There were 628 cases identified for inclusion in the study. Patients undergoing CAO ( $n$ $=44$ ) were more likely to be female or to have higher rates of preoperative spinal drain use, American Society of Anesthesiologists score 23 , low preop hemoglobin, and/or symptomatic presentation, but fewer mean number of aortic zones covered. CAO was associated with higher 30day mortality ( 5 of $44,11 \%$ ) compared to CAP ( 23 of $584,4 \%), P=0.039$. The composite end point occurred at a significantly greater proportion for those who had CAO (10 of 44, 23\%)

Correspondence to: Ryan W. King, MD, Division of Vascular Surgery, Medical University of South Carolina, 30 Courtenay Drive, MSC 295, Charleston, SC 29425, USA; kingry@ musc.edu.

Disclosure: The authors of this manuscript have nothing to disclose. 
compared to CAP (53 of 584, 9\%, $P=0.008$ ), driven by higher rates of 30-day mortality and bowel ischemia (9\% vs. $2 \%, P=0.026$ ). By multivariate analysis, CAO was predictive of 30-day mortality (odds ratio $[\mathrm{OR}]=3.9,95 \%$ confidence interval $[\mathrm{CI}]=1.1-13.8, P=0.04$ ) and the composite endpoint $(\mathrm{OR}=3.0,95 \% \mathrm{CI}=1.1-8.5, P=0.03)$. Increasing procedure time was also associated with 30-day mortality $(\mathrm{OR}=1.4,95 \% \mathrm{CI}=1.1-1.7, P<0.001)$ and the composite end point $(\mathrm{OR}=1.4,95 \% \mathrm{CI}=1.1-1.6, P<0.001)$.

Conclusions: For those treated for TAAAs, CAO was independently predictive of increased 30day mortality and a composite end point of perioperative mortality, spinal cord ischemia, and bowel ischemia. When treating patients with extensive aortic aneurysmal disease, physicians should attempt to preserve the celiac artery, by revascularization or avoiding ostium coverage, whenever feasible.

\section{INTRODUCTION}

Endovascular repair of thoracoabdominal aortic aneurysms (TAAAs) requires consideration of the management of mesenteric and renal vessels. Approximately $15 \%$ of thoracic endovascular aortic repairs (TEVARs) have insufficient distal landing zones ${ }^{1}$ and as many as $4.4 \%^{2}$ to $14 \%^{3}$ of TEVAR cases require celiac artery management due the extent of aortic disease. Failure to achieve recommended distal landing zone can result in type Ib endoleaks, potentiating sac growth. ${ }^{4}$ Current studies suggest that covering the celiac artery during TEVAR is well tolerated given sufficient collateralization from the superior mesenteric artery (SMA). ${ }^{3,5}$ However, compromising celiac artery perfusion may put patients at an additional risk for developing mesenteric ischemia or spinal cord ischemia (SCI) in the postoperative period, in turn, increasing their risk of perioperative mortality. ${ }^{2}$

Most of the available literature suggesting celiac artery occlusion (CAO) during TEVAR is safe and limited to small case series with heterogeneous cohorts inclusive of aneurysm and dissection pathologies. Furthermore, these studies are confounded by SMA and left subclavian artery (LSCA) patency. Associated rates of bowel ischemia following CAO range from $3 \%$ to $11 \%$, while rates of SCI and mortality range from $4 \%$ to $6 \%$ and about $10 \%$, respectively. ${ }^{2,3,6,7}$ Using data obtained through the Society for Vascular Surgery Vascular Quality Initiative (SVS-VQI), the current study is intended to examine the effect of celiac artery occlusion (CAO) versus celiac artery preservation (CAP) on mortality, SCI, and bowel ischemia in patients who underwent endovascular repair for TAAA. Celiac preservation meaning any cases where the celiac artery was revascularized, or coverage of the ostium was avoided. To eliminate potential confounding variables, we selected for nonruptured, TAAA pathology and excluded cases of prior aortic surgeries as well as patients with preoperatively known occlusion of the LSCA, SMA, or celiac arteries.

\section{METHODS}

The SVS-VQI database was queried for TEVAR and complex EVAR cases from May 2012 to May 2018 that fit the following criteria: TAAA pathology, endograft extending to aortic zone 6 (Fig. 1), ${ }^{8}$ excluding cases of aneurysm rupture, patients with acute aneurysms due to trauma, and patients with prior aortic surgery. Due to the known higher risk of visceral ischemia or SCI in patients with known SMA or LSCA occlusion, these patients were 
excluded from analysis. ${ }^{9,10}$ Patients with documented pre-existent CAO were excluded, as no difference in perfusion would be expected with endograft coverage of these patients. The final cohort used for analysis, and excluded cases, are described in an STROBE diagram ${ }^{11}$ (Fig. 2). Cases with intraoperative CAO were compared to cases of CAP. CAP included all cases where the celiac artery was left uncovered or revascularized by open bypass, stent, fenestration, chimney, or scallop techniques. Cases of CAO include all cases in which the celiac artery was occluded (intentionally or unintentionally) by coiling, plugging, or covering with a stent graft. The decision to choose $\mathrm{CAO}$ versus $\mathrm{CAP}$ as comparison groups was to investigate the effects of celiac malperfusion during aortic endovascular repair. It was not possible to determine from the database whether CAO was intentional or inadvertent. Primary outcomes included 30-day mortality and a composite end point of 30-day mortality, SCI, and bowel ischemia. To minimize anatomic heterogeneity, all cases were confirmed to include endograft extension to aortic zone 6 , the origin of the celiac artery. ${ }^{8}$

Bowel ischemia was defined as all cases in which there was endoscopic evidence of ischemia in a patient, bloody stools in a patient who died prior to colonoscopy or laparotomy, or other documented clinical diagnosis. Specific clinical information regarding white blood cell count, fevers, hepatic, and pancreatic laboratory values was not available in VQI; however, these values may have been considered when bowel ischemia was diagnosed in the postoperative period. SCI included all cases of transient and permanent lower extremity neurological deficits following TAAA repair. Myocardial infarction (MI) included all cases listed with postoperative clinical suspicion of MI, electrocardiogram, troponin or cardiac biomarkers, or cardiac imaging findings suggestive of acute MI. Other postoperative variable definitions included the following: dysrhythmia (new cardiac rhythm requiring treatment with cardioversion or medications), congestive heart failure (CHF, new or exacerbation of prior CHF requiring treatment), stroke/transient ischemic attack (TIA, clinical diagnosis of stroke/TIA postoperatively), respiratory complication (pneumonia or reintubation), dialysis (temporary or permanent), and renal ischemia (requiring medical or interventional treatment).

Univariable comparisons were done using chisquared or Fisher's exact tests and Student's $t$ tests, as appropriate. Variables with $P<0.10$ following univariable analyses were included in a stepwise binary logistic regression analysis to identify independent predictors of the primary outcomes. Multivariable analyses were not performed on individual outcomes for SCI and bowel ischemia due to concern for low event rates. Variables were included in the multivariable analyses if $<40 \%$ of cases had missing data. Multicollinearity was accounted for by limiting inclusion in the multivariable model to variables with a Pearson's correlation coefficient value $<0.6$ or a variance inflation factor $<2$. SPSS Statistics Version 24.0 (Armonk, NY: IBM Corporation, 2016) was used for analyses. A 2-tailed $P$-value of $<0.05$ was considered statistically significant. This study was reviewed by the Institutional Review Board of the Medical University of South Carolina, and due to the use of deidentified information, obtaining informed consent was not necessary. 


\section{RESULTS}

From a total of 10,455 patients with TEVAR or complex EVAR in the SVS-VQI database, there were 628 cases identified for inclusion in the study. Among these, the celiac artery was occluded during the procedure in 44 patients (7\%). Demographic, medication-related, anatomic, and periprocedural data were compared between those with CAO and CAP (Tables I and II). Patients undergoing CAO were more likely to be female, to have higher rates of preoperative spinal drain use, American Society of Anesthesiologists ASA scores $\geq 3$, low preop hemoglobin, and symptomatic presentation, but fewer mean number of aortic zones covered compared. In cases of CAP, $60 \%$ were preserved by endovascular means, $5 \%$ had open surgical bypasses, and 35\% had no intervention (endograft landed in aortic zone 6 , but distal to celiac ostium). Outcomes and end points are displayed in Table III. CAO was associated with higher 30 -day mortality ( 5 of $44,11 \%$ vs. 23 of $584,4 \% ; P=0.039$ ).

Recorded data on 30-day all-cause mortality were limited, but all CAO deaths with recorded data showed that death was related to aortic disease, while $11 \%$ of deaths in those with CAP were not related to aortic disease. Bowel ischemia was associated with increased mortality, with 17 cases of bowel ischemia, including 7 leading to patient death (41\%), $P<0.001$. It is worth noting, none of the 4 patients with bowel ischemia and CAO had attempted revascularization: 2 of them had bowel resected and 2 were managed medically. Of the 13 who experienced bowel ischemia with CAP, 3 had attempted revascularizations, 4 had bowel resections, and 6 were managed medically. The mean postoperative length of stay for those who had bowel ischemia was $14.5 \pm 14.4$ days compared to those who did not have bowel ischemia ( $6.2 \pm 6.6$ days), $P=0.008$. Intensive care unit stay was significantly longer for those who had bowel ischemia ( $8.5 \pm 11.1$ days) compared to those who did not $(3.0 \pm 4.2$ days), $P=0.001$. The composite end point occurred at a significantly greater proportion for those who had CAO (10 of $44,23 \%$ ) compared to CAP (53 of 584, 9\%, $P=0.008$ ), driven by higher rates of 30 -day mortality and bowel ischemia ( $9 \%$ vs. $2 \%, P=0.026$ ). CAO was also associated with a longer ICU stay $(5.0 \pm 5.5$ vs. $3.0 \pm 4.5, P<0.001)$, along with significantly more respiratory complications and vasopressor use.

On multivariable analysis, 5 independent predictors of 30-day mortality were identified (Table IV): total procedure time (odds ratio [OR] 1.4, 95\% confidence interval [CI] 1.1-1.7, $P<0.001)$, estimated blood loss $>1,500 \mathrm{cc}(\mathrm{OR} 26.0,95 \% \mathrm{CI}=2.2-308.4, P=0.01)$, intraoperative $\mathrm{CAO}(\mathrm{OR}=3.9,95 \% \mathrm{CI}=1.1-13.8, P=0.04)$, preop hemoglobin $(\mathrm{OR}=0.8$, $95 \% \mathrm{CI}=0.6-1.0, P=0.04$ ), and estimated glomerular filtration rate (eGFR, $\mathrm{OR}=0.98$, $95 \% \mathrm{CI}=0.96-1.0, \mathrm{P} 0.049)$. Five variables were predictive of the composite end point (Table V): total procedure time $(\mathrm{OR}=1.4,95 \% \mathrm{CI}=1.1-1.6, P<0.001)$, chronic obstructive pulmonary disease (COPD; $\mathrm{OR}=2.9,95 \% \mathrm{CI}=1.5-5.7, P<0.001)$, intraoperative $\mathrm{CAO}(\mathrm{OR}=1.4,95 \% \mathrm{CI}=1.1-1.6, P<0.001)$, preop hemoglobin $(\mathrm{OR}=0.8$, $95 \% \mathrm{CI}=0.7 \mathrm{e} 1.0, \mathrm{P} 0.047)$, and number of aortic zones covered $5-6(\mathrm{OR}=2.4,95 \% \mathrm{CI}=$ $1.0-5.5, P=0.049$ ). Importantly, symptomatic presentation, which was significantly higher in the $\mathrm{CAO}$ group at baseline, was not predictive of either 30-day mortality or the composite end point. Specific comorbidities and characteristics for those who experienced an adverse outcome are shown in Table VI.

Ann Vasc Surg. Author manuscript; available in PMC 2020 July 01. 


\section{DISCUSSION}

The celiac artery provides perfusion to many vital organs, and literature describes potential complications related to CAO during aortic aneurysm repair. The celiac artery perfuses the liver, gallbladder, spleen, and foregut via the common hepatic, splenic, and left gastric arteries. ${ }^{12}$ The SMA contributes collateral perfusion to the celiac artery distribution primarily through the pancreaticoduodenal arcade (inclusive of the gastroduodenal artery) and the dorsal pancreatic artery. ${ }^{13}$ Occlusion or ligation of the celiac artery can subsequently lead to ischemic pancreatitis, ${ }^{14}$ hepatitis, ${ }^{3,6}$ cholangiopathy, ${ }^{3}$ and acute or chronic bowel ischemia. ${ }^{15} \mathrm{SCI}$ becomes a concern during $\mathrm{CAO}$ due to endograft coverage at spinal levels T12-L1, ${ }^{16}$ potentially compromising collateral spinal perfusion via the artery of Adamkiewicz at T10-L2 ${ }^{17}$ and intercostal arteries, which branch along the thoracic aorta. Unlike the hypogastric and subclavian arteries, the celiac artery is not known for supplying the spinal cord. ${ }^{18}$ As many as $4.4 \%^{2}$ to $14 \%^{3}$ of TEVAR cases require celiac artery management due the extent of aortic disease. CAO can be performed by applying embolization techniques and/or by excluding the ostium with endograft coverage, while CAP can be performed by open bypass or by endovascular techniques such as fenestrated, snorkel, scallop, and by adding a sidearm graft to a traditional endograft. Approximately half of those who had CAP within this VQI cohort had endovascular celiac artery management (60\%) while only 5\% had open bypass. About 35\% of the CAP group had endograft extension into aortic zone 6 , but preserved the celiac artery ostium by landing the endograft distally.

Most prior studies suggest that CAO during endovascular aortic repair is generally well tolerated, but these studies are limited by small sample sizes and/or comparisons of heterogenous groups. ${ }^{6,19-21}$ In a review of 31 patients undergoing $\mathrm{CAO}$, the rates of visceral ischemia, SCI, and death were all $6 \%{ }^{3}$ Similarly, Rose et al. ${ }^{6}$ reported on 18 patients (of 366 over 9 years) who had $\mathrm{CAO}$ and 2 had bowel ischemia with an additional patient having symptoms of chronic mesenteric ischemia with weight loss, and 2 had SCI. In a singlecenter experience from 2000 to 2006, 16 of 434 patients had CAO, leading to 3 deaths, 4 cases of bowel ischemia, and 2 cases of SCI; 3 patients had celiac artery revascularization with no ischemic complications. ${ }^{2}$ Smaller case series of $<10$ patients have outcomes free of ischemic events, ${ }^{22,23}$ while other small reports observed ischemic hepatitis ${ }^{24}$ and intestinal angina after CAO. ${ }^{21}$ In a larger 2013 literature review of 93 total CAO cases inclusive of various aortic pathologies, the 30-day mortality rate was $9.7 \%$, with $8.6 \%$ of patients having had complications related to foregut ischemia $(n=8)$, and 5.4\% developed SCI $(n=5){ }^{7}$

Delineating the potential risks of occluding the celiac artery during TEVAR necessitates accounting for variables that may have confounded results of prior studies, including LSCA and SMA patency, type of aortic pathology, and anatomic location of endograft placement, which our analysis provides. By eliminating all cases of SMA and LSCA occlusion in the current study, an attempt was made to minimize cases of bowel ischemia and SCI caused by compromised branches other than celiac artery. ${ }^{9,10}$ Cases of trauma and ruptures are associated with higher mortality rates secondary to mechanism and can be more likely to involve celiac artery coverage as urgency limits time for planned branch preservation. ${ }^{25}$ Prior abdominal aortic repairs have been shown to increase the risk of SCI, and may also 
confound bowel ischemia outcomes, and were thus excluded. ${ }^{26}$ Higher rates of SCI and bowel ischemia have also been reported for aortic dissections compared to aneurysms. ${ }^{27}$ This study stands as an analysis of a more limited group, attempting to identify mortality and morbidity outcomes likely attributable to the management of the celiac artery during elective TEVAR for TAAA.

Overall mortality in this study's VQI cohort approximated 4\%, with a significant difference between those with CAO $(11 \%)$ and with CAP (4\%). The exact etiology of the perioperative death could not be determined from the VQI. However, all reported cases of 30-day mortality within the $\mathrm{CAO}$ group were related to the aortic disease process, and CAO was associated with an almost 4 times increased risk of 30-day mortality. Other identified risk factors such as increasing total procedure time, estimated blood loss $>1,500 \mathrm{cc}$, low preop hemoglobin, and low eGFR were not unexpected predictors as supported by the current literature. Risk factors for mortality after TEVAR in literature include persistent type I or III endoleaks, emergent repair, postop MI or neurologic injury, 28 age $>80$, ASA class $>3$, elevated serum creatinine, $>4$ units transfused preop, and elevated white blood cell counts. ${ }^{29}$ Symptomatic presentation was not found to be predictive of 30-day mortality in this cohort. To our knowledge, no other studies have identified CAO as an independent predictor of perioperative mortality.

Beyond identified risk factors for 30-day mortality in this study, bowel ischemia was higher in the CAO group and found to be associated with increased mortality. This may suggest that coverage of the celiac artery intraoperation may increase the risk of inducing bowel ischemia, subsequently leading to the higher mortality rates observed in the CAO group. An acute cause of death after $\mathrm{CAO}$, such as bowel ischemia, is suggested by the nonsignificant difference in 1-year mortality between the 2 groups. Mehta et al. ${ }^{3}$ who reported a $6 \%$ rate of bowel ischemia and 6\% rate of SCI did not show any further events at a 15-month follow up among their patients, supporting most adverse outcomes related to CAO occur acutely postop. The associated mortality with bowel ischemia after endovascular aortic repair is as high as $50 \% .^{30}$ The incidence of bowel ischemia observed in CAO cases (9\%) was significantly higher than those with CAP (2\%). This difference could be attributable to CAO for several reasons: (1) there is only a $0.5-3 \%$ incidence of bowel ischemia after infrarenal EVAR in which the inferior mesenteric artery (IMA) is excluded, ${ }^{31-33}$ (2) all CAOs occurred acutely intraoperation, (3) only cases with patent SMAs were selected, and (4) the overall incidence of ischemic complications related to CAO may have been underestimated in this study, because data on hepatic and gallbladder pathologies were not available. CAO may put patients at risk for bowel ischemia and subsequent death.

The significantly higher rate of the composite end point (30-day mortality, bowel ischemia, and SCI) observed with CAO was primarily driven by differences in 30-day mortality and bowel ischemia, but not SCI. SCI was not significantly different between the comparison groups, which is consistent with the lack of arterial branches perfusing the spinal cord from the celiac artery. We selected cases with extension into aortic zone 6, thus similar parts of the spinal cord were covered, such any discrepancy in SCI outcomes due to coverage of the artery of Adamkiewicz or the intercostal arteries at this level would have been accounted for. We were, however, able to demonstrate that the number of aortic zones covered was 
predictive of the composite end point and possibly related to increased SCI with more aortic coverage, which has been shown as a risk factor for SCI. ${ }^{34}$ Overall, risk factors for the composite end point were similar to the risk factors identified for 30-day mortality: total procedure time, $\mathrm{CAO}$, low preop hemoglobin, $\mathrm{COPD}$, and the number of aortic zones covered. Symptomatic presentation, which was significantly higher in the CAO group (34\% vs. $12 \%$ ), was not predictive of 30-day mortality or the composite end point in this VQI cohort, which may reflect improvement in outcomes for symptomatic aneurysm repairs in contemporary times, suggested by De Martino et al. ${ }^{35}$ who demonstrated that in-hospital mortality was similar for those had symptomatic aneurysm (1.3\%) repairs compared to those who had elective repairs (1.7\%).

Despite the conservative analysis presented, there are multiple limitations to this study to note. Some studies suggest that preoperative evaluation of the established SMA-celiac artery collaterals be performed by angiography prior to $\mathrm{CAO},{ }^{3,7,22}$ and although we could select cases based on preop patency of branch vessels, VQI did not collect data on established collaterals. Furthermore, information on the IMA and hypogastric artery patency was not available and it is possible that some of our TEVAR cases extended down to exclude the IMA, but this was difficult to determine given aortic zone 9 includes the entire infrarenal aorta. We were unable to report exact aortic coverage lengths, but used aortic zones as a surrogate. Determining the exact cause of mortality was limited, and most cases of 30-day mortality did not have a specific cause of death. Complications related to liver, gallbladder, or pancreatic pathology were not available and some clinical information was not included in the VQI database, including white blood cell count, fevers, hepatic, and pancreatic laboratory values. It was also not possible to determine among cases with $\mathrm{CAO}$ whether continued perfusion of the celiac axis was even feasible. Our CAO group only included 44 cases compared to 584 cases in the CAP group. Finally, this series represents a retrospective analysis of a prospective database and is thus subject to standard reporting biases, coding errors, and missing data.

\section{CONCLUSION}

Using the VQI to obtain a relatively large cohort, we were able to eliminate important confounding variables and compare outcomes of those who had CAO versus CAP during TEVAR for TAAA. CAO was predictive of an increase in 30-day mortality and a composite end point of 30-day mortality, SCI, and bowel ischemia. CAO correlated with postoperative bowel ischemia, which was also associated with increased mortality. Physicians should preserve the celiac artery during endovascular aortic repair when feasible, as CAO may predispose patients to higher mortality and bowel ischemia.

\section{REFERENCES}

1. Jackson BM, Carpenter JP, Fairman RM, et al. Anatomic exclusion from endovascular repair of thoracic aortic aneurysm. J Vasc Surg 2007;45:662-6. [PubMed: 17350215]

2. Leon LR Jr, Mills JL, Jordan W, et al. The risks of celiac artery coverage during endoluminal repair of thoracic and thoracoabdominal aortic aneurysms. Vasc Endovascular Surg 2009;43:51-60. [PubMed: 18996912] 
3. Mehta M, Darling RC 3rd, Taggert JB, et al. Outcomes of planned celiac artery coverage during TEVAR. J Vasc Surg 2010;52:1153-8. [PubMed: 20709480]

4. Nation DA, Wang GJ. TEVAR: endovascular repair of the thoracic aorta. Semin Intervent Radiol 2015;32:265e71. [PubMed: 26327745]

5. Falkenberg M, Lonn L, Schroeder T, et al. TEVAR and covering the celiac artery. Is it safe or not? J Cardiovasc Surg (Torino) 2010;51:177-82. [PubMed: 20354487]

6. Rose MK, Pearce BJ, Matthews TC, et al. Outcomes after celiac artery coverage during thoracic endovascular aortic aneurysm repair. J Vasc Surg 2015;62:36-42. [PubMed: 25937603]

7. Jim J, Caputo FJ, Sanchez LA. Intentional coverage of the celiac artery during thoracic endovascular aortic repair. J Vasc Surg 2013;58:270-5. [PubMed: 23711693]

8. Fillinger MF, Greenberg RK, McKinsey JF, et al. Reporting standards for thoracic endovascular aortic repair (TEVAR). J Vasc Surg 2010;52:1022-1033.e5. [PubMed: 20888533]

9. Oldenburg WA, Lau LL, Rodenberg TJ, et al. Acute mesenteric ischemia: a clinical review. JAMA Intern Med 2004;164:1054-62.

10. Teixeira PG, Woo K, Beck AW, et al. Association of left subclavian artery coverage without revascularization and spinal cord ischemia in patients undergoing thoracic endovascular aortic repair: a Vascular Quality Initiative(R) analysis. Vascular 2017;25:587-97. [PubMed: 29022855]

11. von Elm E, Altman DG, Egger M, et al. The Strengthening the Reporting of Observational Studies in Epidemiology (STROBE) statement: guidelines for reporting observational studies. J Clin Epidemiol 2008;61:344-9. [PubMed: 18313558]

12. Santos PVD, Barbosa ABM, Targino VA, et al. Anatomic variations of the celiac trunk: a systemic review. Arq Bras Cir Dig 2018;31:e1403. [PubMed: 30539978]

13. Song S-Y, Chung JW, Kwon JW, et al. Collateral pathways in patients with celiac axis stenosis: angiographicespiral CT correlation. Radiographics 2002;22:881-93. [PubMed: 12110717]

14. Kawatani Y, Kurobe H, Nakamura Y, et al. Acute pancreatitis caused by pancreatic ischemia after TEVAR combined with intentional celiac artery coverage and embolization of the branches of the celiac artery. J Surg Case Rep 2017;2017:rjx029. [PubMed: 28458836]

15. Ayad M, Senders ZJ, Ryan S, et al. Chronic mesenteric ischemia after partial coverage of the celiac artery during TEVAR, case report, and review of the literature. Ann Vasc Surg 2014;28:1935.e1-6.

16. Selvaraj L, Sundaramurthi I. Study of normal branching pattern of the coeliac trunk and its variations using CT angiography. J Clin Diagn Res 2015;9:AC01-4.

17. Kudo K, Terae S, Asano T, et al. Anterior spinal artery and artery of Adamkiewicz detected by using multi-detector row CT. Am J Neuroradiol 2003;24:13-7. [PubMed: 12533320]

18. Eagleton MJ, Shah S, Petkosevek D, et al. Hypogastric and subclavian artery patency affects onset and recovery of spinal cord ischemia associated with aortic endografting. J Vasc Surg 2014;59:8994. [PubMed: 24188715]

19. Rao AS, Rhee RY. Coverage of the celiac artery during TEVAR: is it ever appropriate? Semin Vasc Surg 2009;22: 152-8. [PubMed: 19765525]

20. Li M, Shu C, Li Q, et al. Midterm results of intentional celiac artery coverage during TEVAR for type B aortic dissection. J Endovasc Ther 2013;20:276-82. [PubMed: 23731296]

21. Waldenberger P, Bendix N, Petersen J, et al. Clinical outcome of endovascular therapeutic occlusion of the celiac artery. J Vasc Surg 2007;46:655-61. [PubMed: 17764875]

22. Vaddineni SK, Taylor SM, Patterson MA, et al. Outcome after celiac artery coverage during endovascular thoracic aortic aneurysm repair: preliminary results. J Vasc Surg 2007;45:467-71. [PubMed: 17254741]

23. Brinster CJ, Szeto WY, Bavaria JE, et al. Endovascular repair of extent I thoracoabdominal aneurysms with landing zone extension into the aortic arch and mesenteric portion of the abdominal aorta. J Vasc Surg 2010;52: 460-3. [PubMed: 20541342]

24. Delle M, Lonn L, Henrikson O, et al. Celiac trunk coverage in endovascular aneurysm repair. Scand J Surg 2010;99: 226-9. [PubMed: 21159593]

25. Arthurs ZM, Starnes BW, Sohn VY, et al. Functional and survival outcomes in traumatic blunt thoracic aortic injuries: an analysis of the National Trauma Databank. J Vasc Surg 2009;49:98894. [PubMed: 19341888] 
26. Baril DT, Carroccio A, Ellozy SH, et al. Endovascular thoracic aortic repair and previous or concomitant abdominal aortic repair: is the increased risk of spinal cord ischemia real? Ann Vasc Surg 2006;20:188-94. [PubMed: 16550478]

27. Gialdini G, Parikh NS, Chatterjee A, et al. Rates of spinal cord infarction after repair of aortic aneurysm or dissection. Stroke 2017;48:2073-7. [PubMed: 28655811]

28. Czerny M, Funovics M, Ehrlich M, et al. Risk factors of mortality in different age groups after thoracic endovascular aortic repair. Ann Thorac Surg 2010;90:534-8. [PubMed: 20667346]

29. Hu FY, Fang ZB, Leshnower BG, et al. Contemporary evaluation of mortality and stroke risk after thoracic endovascular aortic repair. J Vasc Surg 2017;66:718-727.e5. [PubMed: 28502542]

30. Brewster DC, Franklin DP, Cambria RP, et al. Intestinal ischemia complicating abdominal aortic surgery. Surgery 1991;109:447-54. [PubMed: 1844392]

31. Becquemin JP, Majewski M, Fermani N, et al. Colon ischemia following abdominal aortic aneurysm repair in the era of endovascular abdominal aortic repair. J Vasc Surg 2008;47:258-63. discussion 63. [PubMed: 18241745]

32. Miller A, Marotta M, Scordi-Bello I, et al. Ischemic colitis after endovascular aortoiliac aneurysm repair: a 10-year retrospective study. Arch Surg 2009;144:900-3. [PubMed: 19841356]

33. Maldonado TS, Rockman CB, Riles E, et al. Ischemic complications after endovascular abdominal aortic aneurysm repair. J Vasc Surg 2004;40:703-9. discussion 9-10. [PubMed: 15472598]

34. Feezor RJ, Martin TD, Hess PJ Jr, et al. Extent of aortic coverage and incidence of spinal cord ischemia after thoracic endovascular aneurysm repair. Ann Thorac Surg 2008;86: 1809-14. discussion 14. [PubMed: 19021982]

35. De Martino RR, Nolan BW, Goodney PP, et al. Outcomes of symptomatic abdominal aortic aneurysm repair. J Vasc Surg 2010;52:5-12.e1. [PubMed: 20471771] 

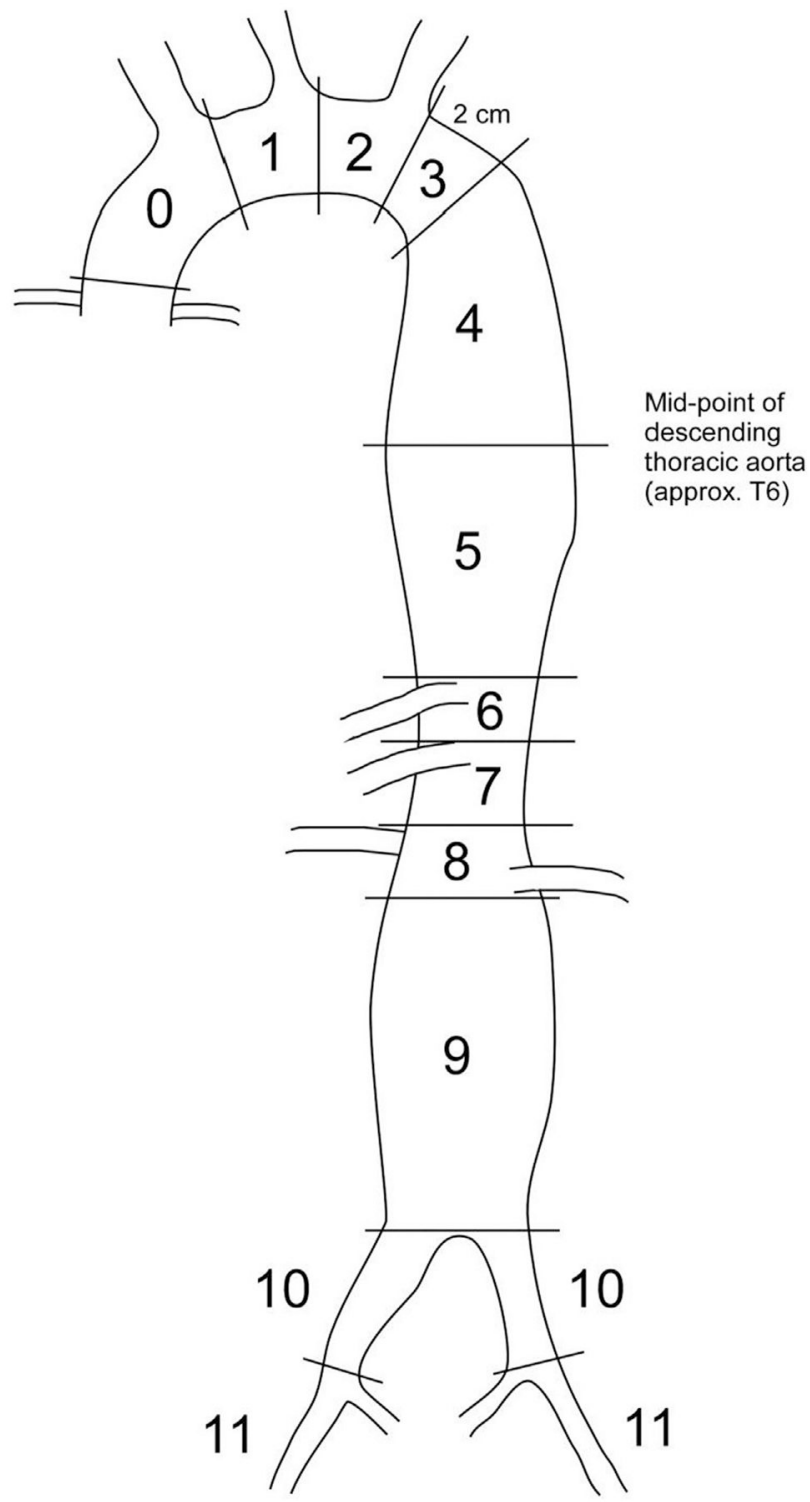

Fig. 1.

Aortic zones described by the Society for Vascular Surgery reporting standards for TEVAR. Aortic zones included are $0-5$. Zone 6 begins at the celiac origin and extends to the superior mesenteric artery. ${ }^{8}$ 


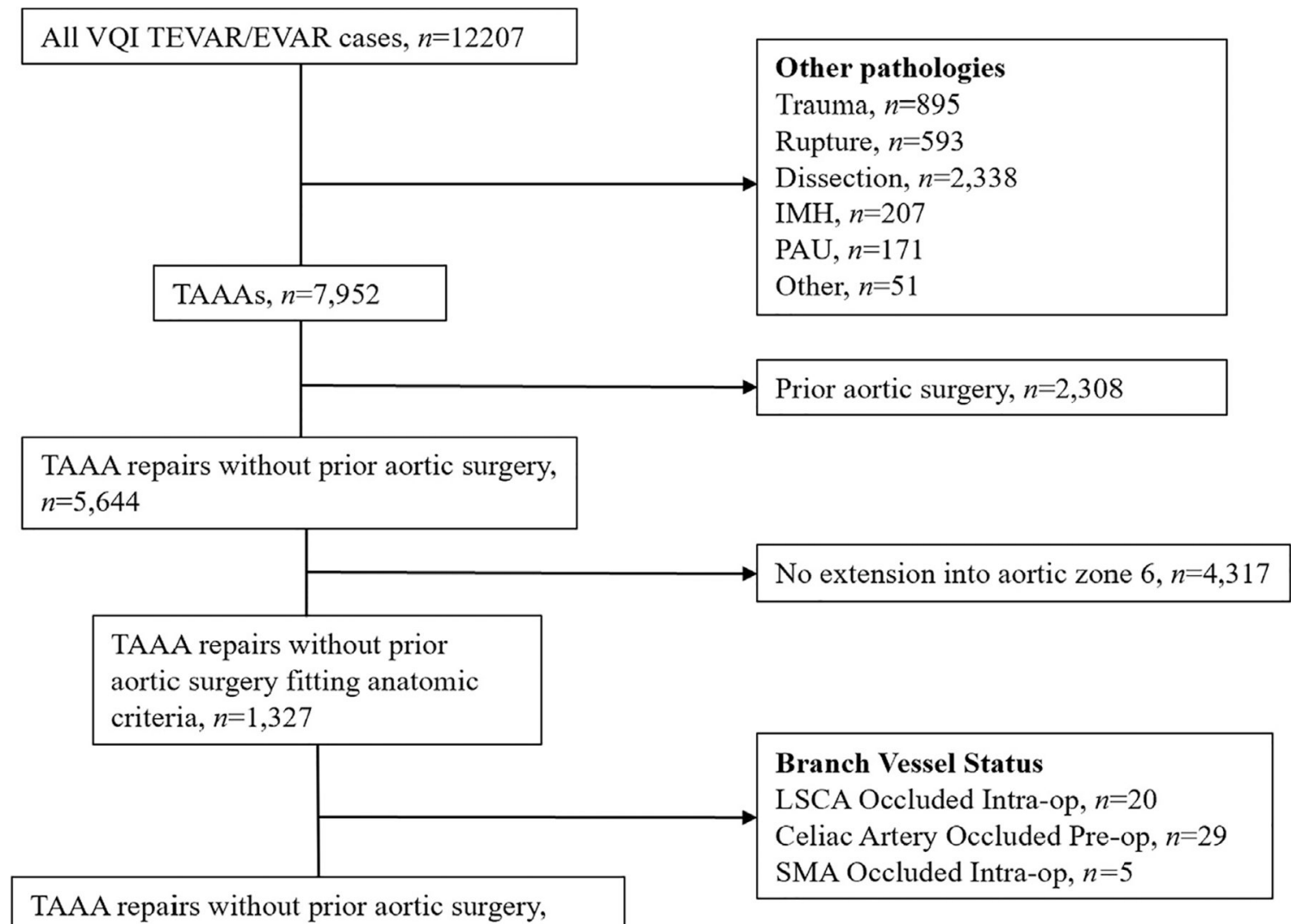

fitting anatomic criteria, without confounding branch vessels, $n=1,273$

\begin{tabular}{|l|l|l|} 
& & $\begin{array}{l}\text { Missing Data } \\
\text { SCI data missing, } n=10 \\
\text { SMA data missing, } n=116 \\
\text { Death data missing, } n=519\end{array}$ \\
\cline { 2 - 3 } & Final cohort, TAAAs without prior &
\end{tabular}

repairs, fitting anatomic criteria, minimal missing data, $n=628$

Fig. 2.

STROBE diagram demonstrating the final cohort used for analysis and the excluded cases. Aortic zone 6 includes the origin of the celiac artery. ${ }^{8} \mathrm{IMH}$, intramural hematoma; PAU, penetrating aortic ulcer. 


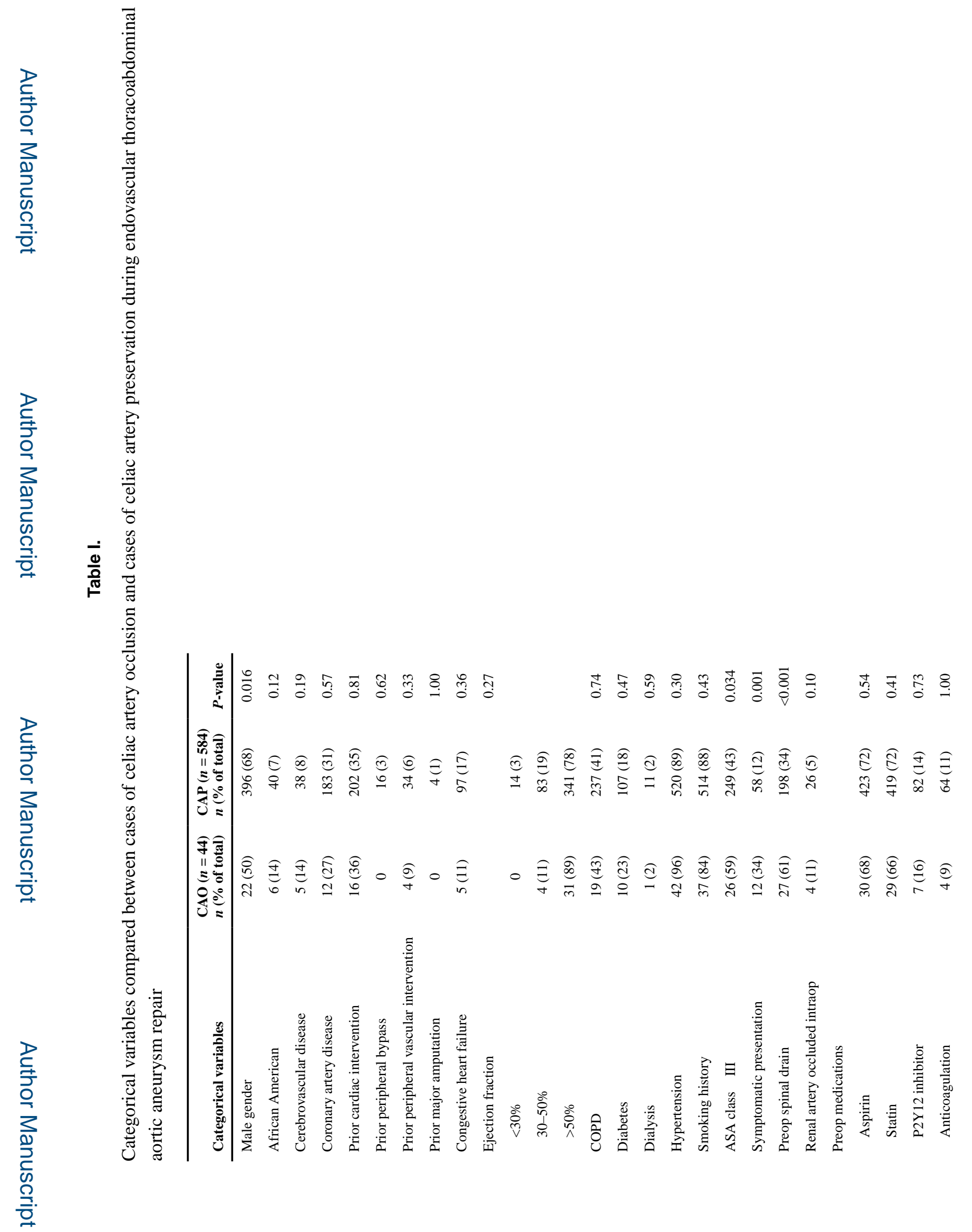

Ann Vasc Surg. Author manuscript; available in PMC 2020 July 01. 


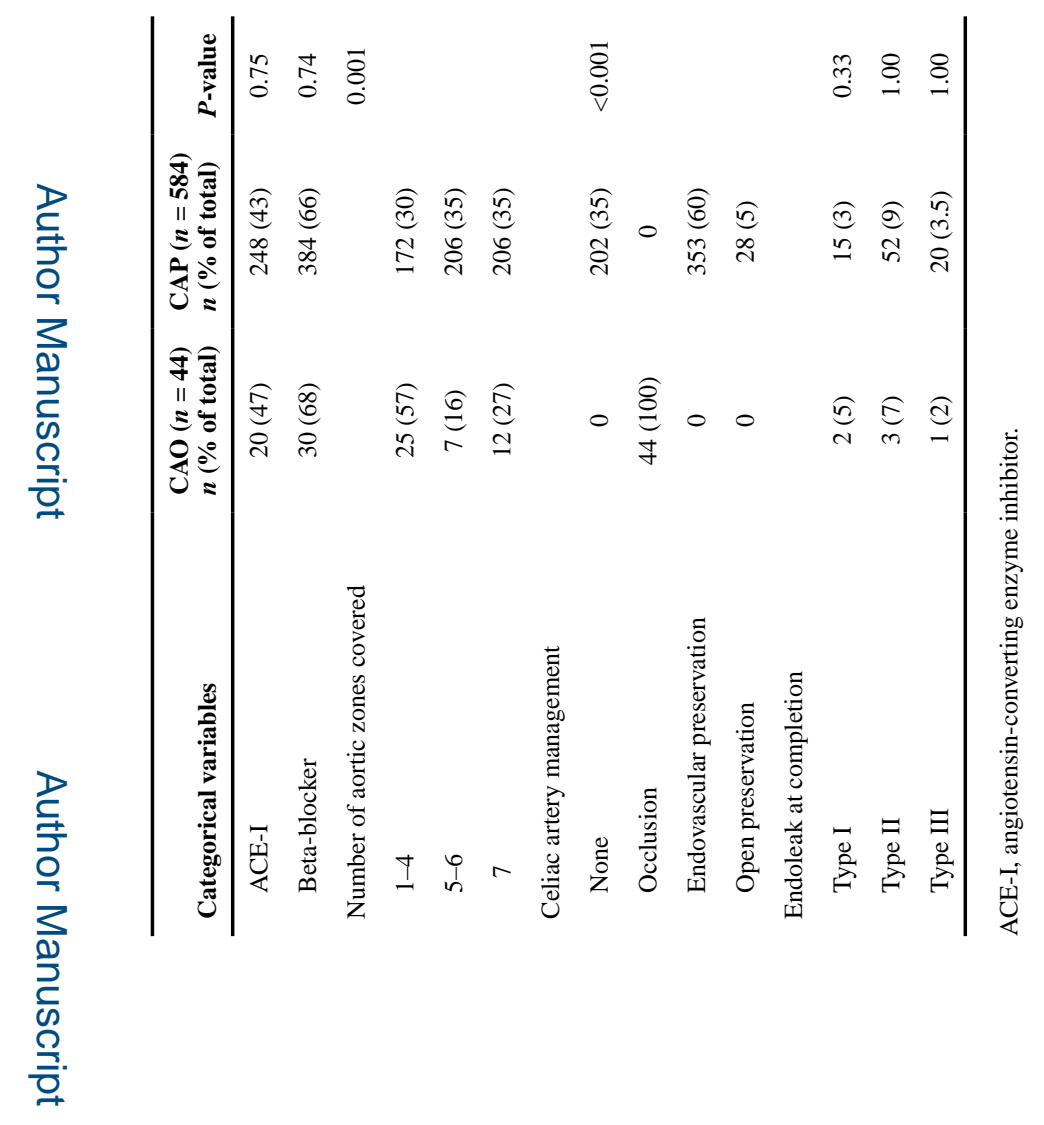

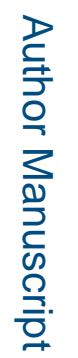

를

Ann Vasc Surg. Author manuscript; available in PMC 2020 July 01. 


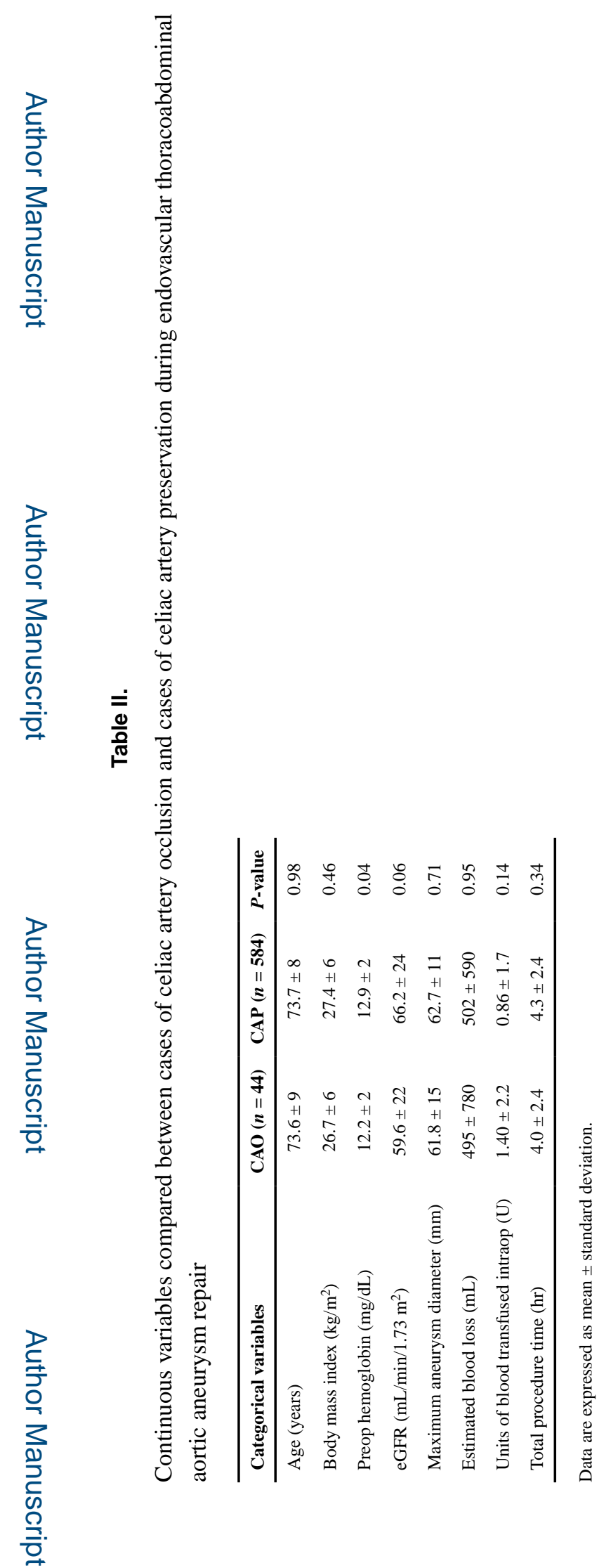

Ann Vasc Surg. Author manuscript; available in PMC 2020 July 01. 


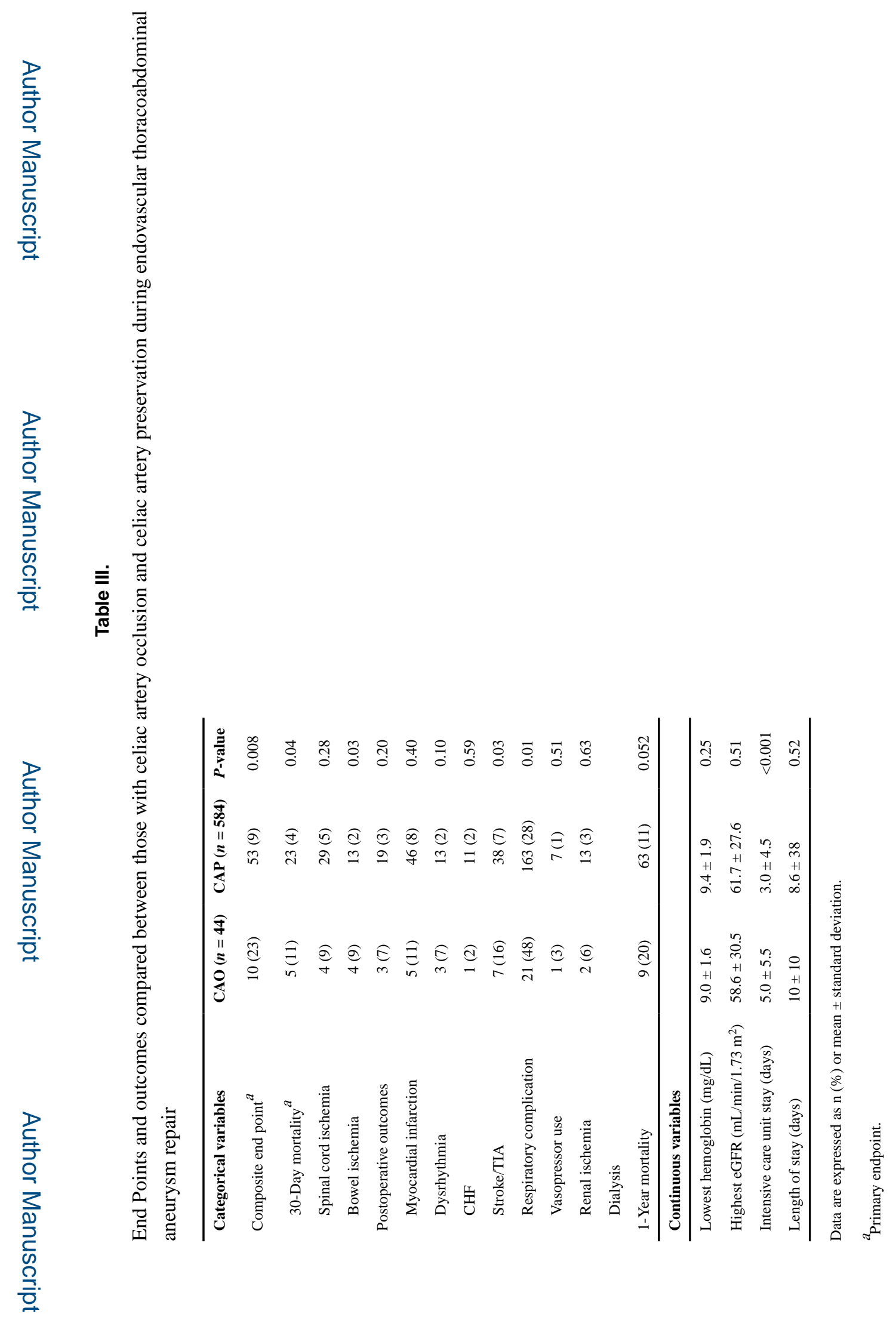

Ann Vasc Surg. Author manuscript; available in PMC 2020 July 01. 


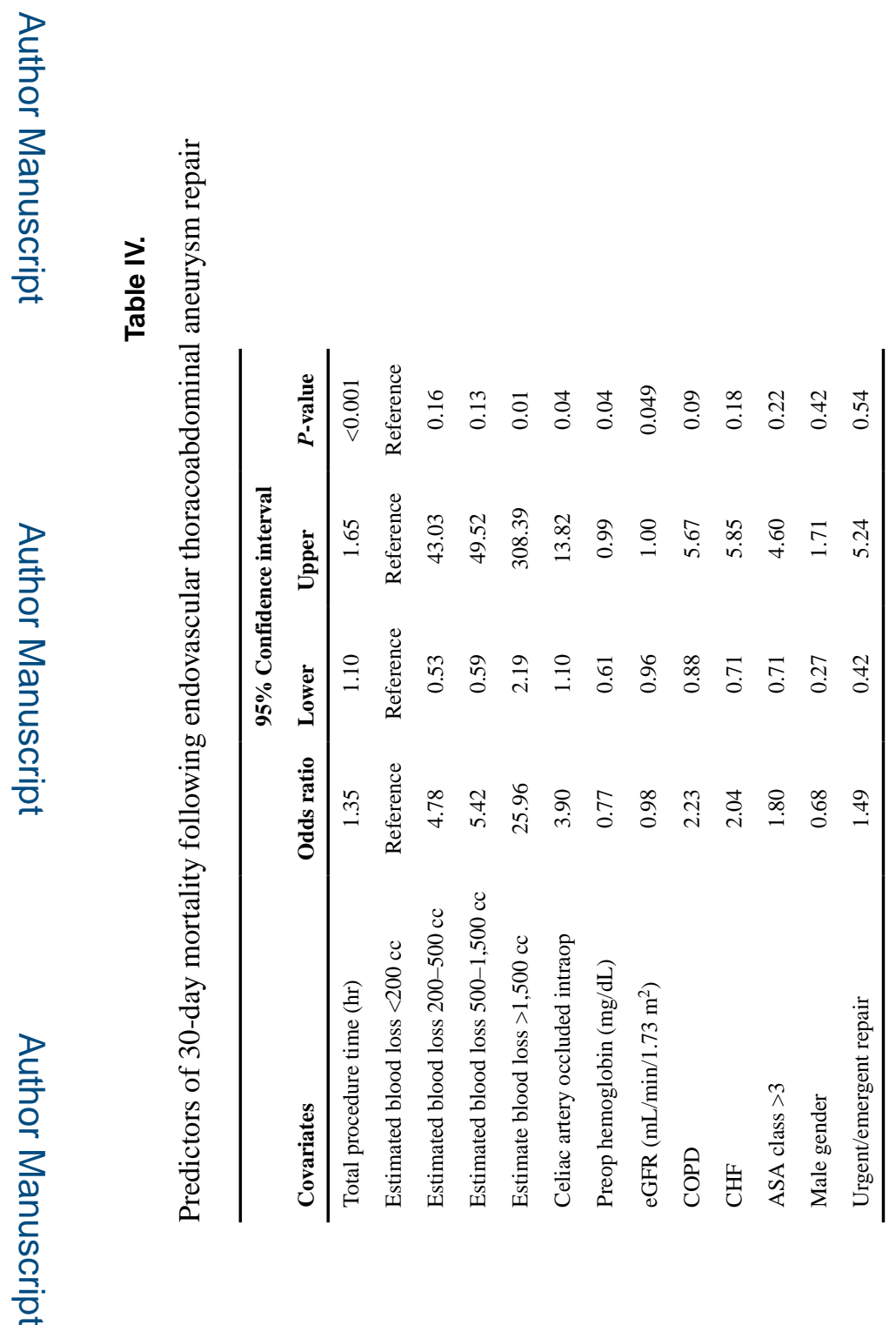

Ann Vasc Surg. Author manuscript; available in PMC 2020 July 01. 


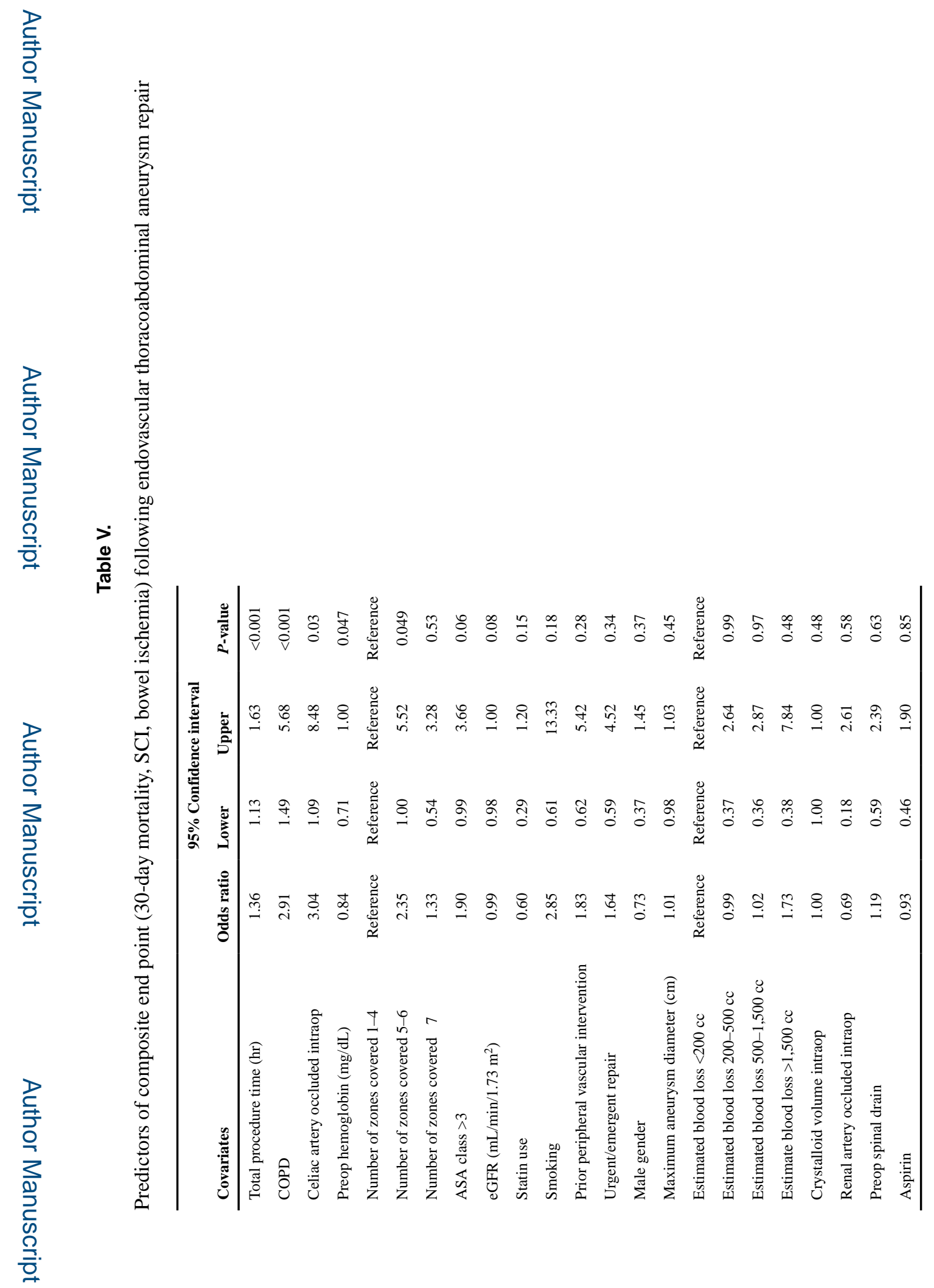

Ann Vasc Surg. Author manuscript; available in PMC 2020 July 01. 


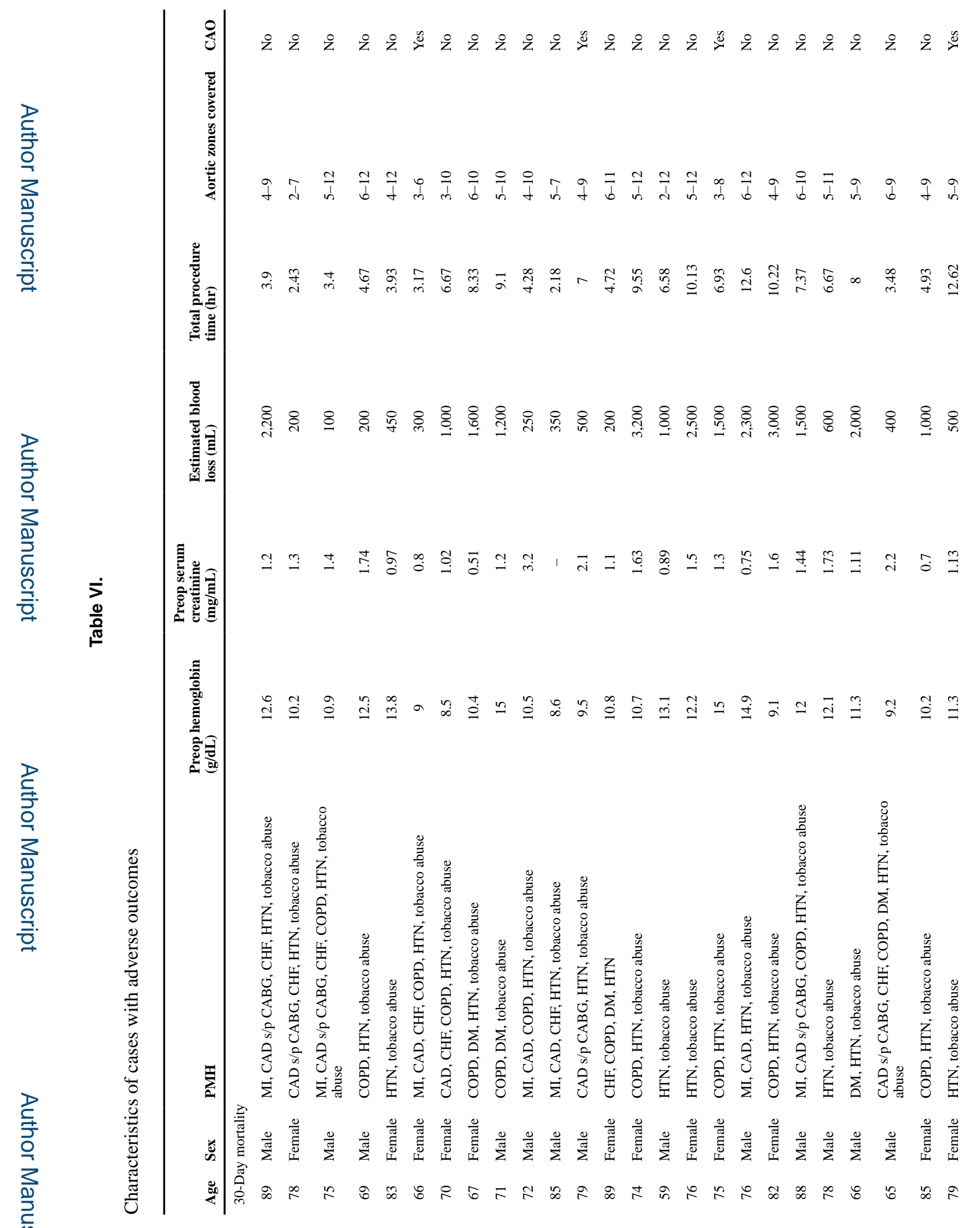

Ann Vasc Surg. Author manuscript; available in PMC 2020 July 01. 


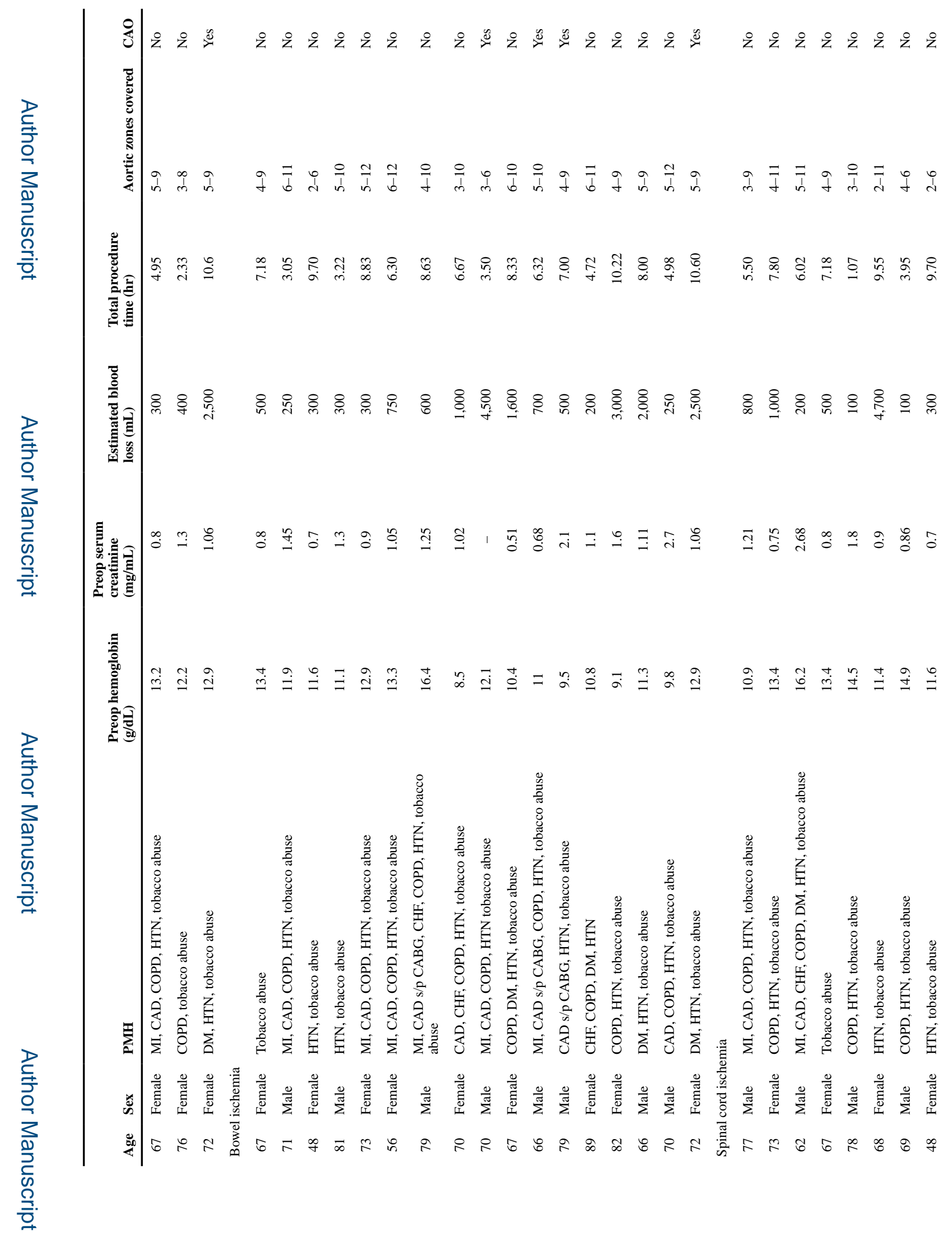

Ann Vasc Surg. Author manuscript; available in PMC 2020 July 01. 


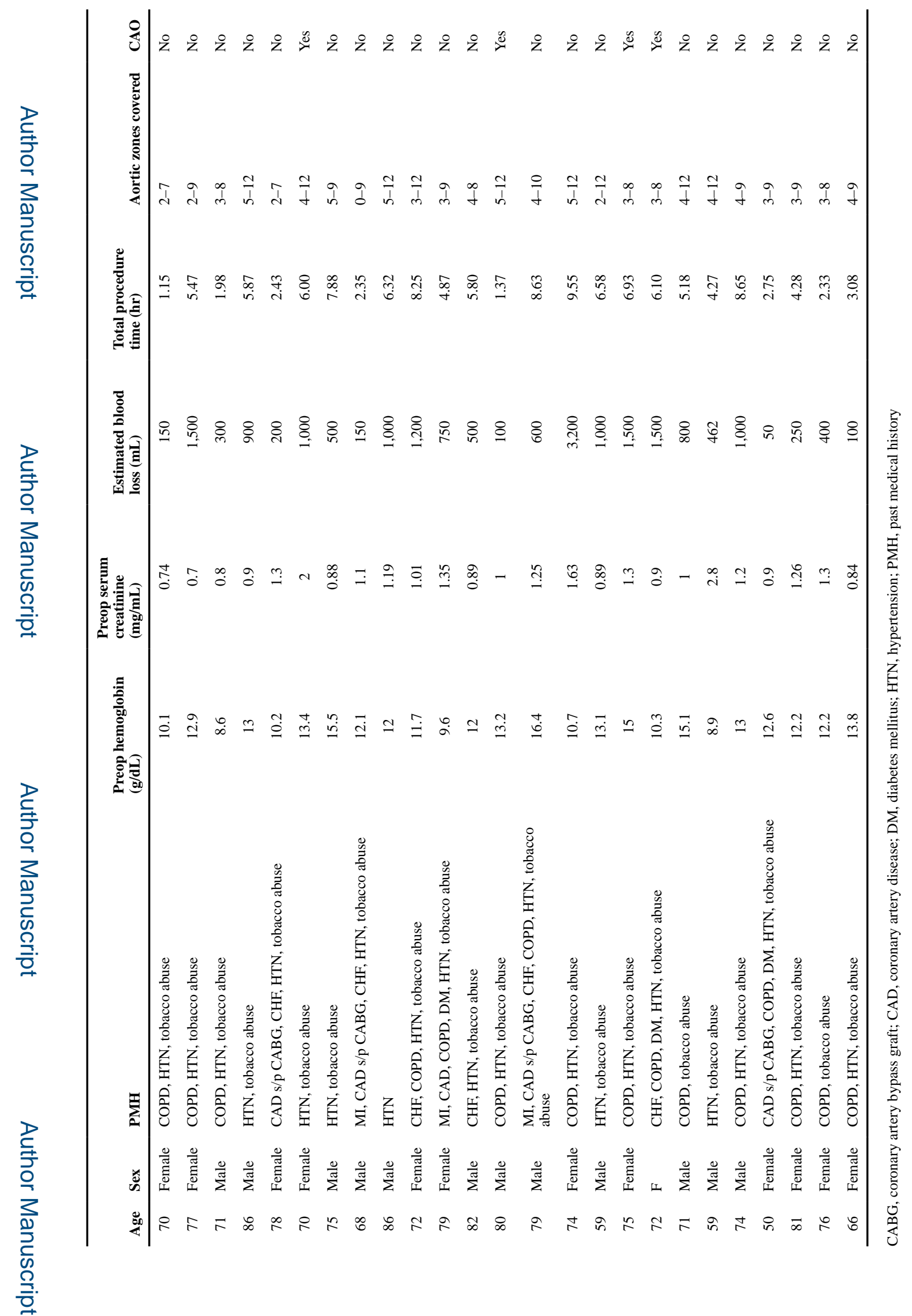

Ann Vasc Surg. Author manuscript; available in PMC 2020 July 01. 